\title{
Chemogenetic Inactivation of Dorsal Anterior Cingulate Cortex Neurons Disrupts Attentional Behavior in Mouse
}

\author{
Hiroyuki Koike ${ }^{1,2,3,4,5}$, Michael P Demars ${ }^{1,2,3,4,5}$, Jennifer A Short ${ }^{1,2,3,4,5}$, Elisa M Nabel ${ }^{1,2,3,4,5}$, \\ Schahram Akbarian ${ }^{1,2,5}$, Mark G Baxter ${ }^{2,5}$ and Hirofumi Morishita ${ }^{*, 1,2,3,4,5}$ \\ 'Department of Psychiatry, Icahn School of Medicine at Mount Sinai, New York, NY, USA; '2Department of Neuroscience, Icahn School of Medicine \\ at Mount Sinai, New York, NY, USA; ${ }^{3}$ Department of Ophthalmology, Icahn School of Medicine at Mount Sinai, New York, NY, USA; ${ }^{4}$ Mindich Child \\ Health and Development Institute, Icahn School of Medicine at Mount Sinai, New York, NY, USA; ${ }^{5}$ Friedman Brain Institute, Icahn School of \\ Medicine at Mount Sinai, New York, NY, USA
}

\begin{abstract}
Attention is disrupted commonly in psychiatric disorders, yet mechanistic insight remains limited. Deficits in this function are associated with dorsal anterior cingulate cortex (dACC) excitotoxic lesions and pharmacological disinhibition; however, a causal relationship has not been established at the cellular level. Moreover, this association has not yet been examined in a genetically tractable species such as mice. Here, we reveal that dACC neurons causally contribute to attention processing by combining a chemogenetic approach that reversibly suppresses neural activity with a translational, touchscreen-based attention task in mice. We virally expressed inhibitory hM4Di DREADD (designer receptor exclusively activated by a designer drug) in $\mathrm{dACC}$ neurons, and examined the effects of this inhibitory action with the attention-based five-choice serial reaction time task. DREADD inactivation of the dACC neurons during the task significantly increased omission and correct response latencies, indicating that the neuronal activities of dACC contribute to attention and processing speed. Selective inactivation of excitatory neurons in the AACC not only increased omission, but also decreased accuracy. The effect of inactivating $\mathrm{dACC}$ neurons was selective to attention as response control, motivation, and locomotion remain normal. This finding suggests that dACC excitatory neurons play a principal role in modulating attention to task-relevant stimuli. This study establishes a foundation to chemogenetically dissect specific cell-type and circuit mechanisms underlying attentional behaviors in a genetically tractable species. Neuropsychopharmacology (2016) 4I, I014-1023; doi: I0.1038/npp.2015.229; published online 26 August 2015
\end{abstract}

\section{INTRODUCTION}

Attention deficits are commonly observed across several psychiatric conditions, including schizophrenia (Chen and Faraone, 2000; Cornblatt and Malhotra, 2001), attention deficit hyperactivity disorder (Huang-Pollock et al, 2012), and several mood disorders (Brown et al, 1994). Although the neurobiological basis of attention has been heavily investigated, the regions implicated in these studies have not yet been tested for their mechanistic casual contributions. A genetically tractable animal model, particularly in a thoroughly examined species such as mouse, could test the causal contribution of neuronal activity to attentional behavior. In turn, an attention task in mice that has been highly characterized on a cellular basis could facilitate diagnosis, prevention, treatment, and cure of deficits in psychiatric disorders.

Mechanistic neural contributions to behavior can be addressed using engineered $G$ protein-coupled receptors known as DREADDs (designer receptors exclusively activated

* Correspondence: Professor $\mathrm{H}$ Morishita, Department of Psychiatry, Icahn School of Medicine at Mount Sinai, One Gustave L Levy Place, Box 1230, New York, NY 10029, USA, Tel: + I 212824 8975, Fax: + I 646537 9584, E-mail: hirofumi.morishita@mssm.edu

Received 22 May 2015; revised 25 July 20I5; accepted 28 July 20I5; accepted article preview online 30 July 2015 by designer drugs) by providing chemogenetic means to achieve a high level of temporal, reversible, and noninvasive manipulation of neuronal activity (Zhu and Roth, 2014). The utility of this approach is substantiated in accumulating studies that have applied technology to specific cell types and neural circuits in the context of behavioral assays of brain function following its inception (Urban and Roth, 2015). Human M4 muscarinic DREADD receptor coupled to Gi (hM4Di) has been used to silence neuronal firing in vivo (Ferguson et al, 2011; Krashes et al, 2011), and has successfully elucidated neural mechanisms mediating complex cognitive processes, such as goal-directed behavior (Parnaudeau et al, 2013; Parnaudeau et al, 2015). This powerful chemogenetic tool has not been employed to examine the neural basis of attention processes. Establishing a chemogenetic manipulation in conjunction with an attention task could advance understandings of the neural basis of attention processing.

Accumulating evidence across species points to a conserved role of the dorsal anterior cingulate cortex (dACC), a dorsal part of medial prefrontal cortex, in normal attention processing. Cytoarchitectural similarities across human, primate, and rodent dACC are accompanied by shared functional findings (Uylings et al, 2003; Vogt and Paxinos, 2014). Observational studies indicate that the dACC selectively responds to task-relevant stimuli. Human studies 
show that dACC activity correlates with performance when study participants attend to task-relevant stimuli (Alexander et al, 2005; Corbetta et al, 1991; Weissman et al, 2005). Furthermore, the dACC is hypoactivated in patients with attention-deficit/hyperactivity disorder during an attention task (Bush et al, 1999). Shrinkage of the dACC that is commonly observed in multiple psychiatric disordersincluding schizophrenia, bipolar disorder, depression, addiction, obsessive-compulsive disorder, and anxiety-correlates with poor executive functioning including sustained attention (Goodkind et al, 2015). Electrophysiologic singleunit recording in both primates and rodents also demonstrate dACC activity during attention tasks, specifically in anticipation of oncoming task-relevant visual cues (Johnston et al, 2007; Totah et al, 2009). These observations are corroborated by lesion observations. In humans, anterior cingulotomy produces attentional deficits (Alexander et al, 2005; Cohen et al, 1999; Ochsner et al, 2001). In rodents, both excitotoxic lesions and pharmacologic GABAergic disinhibition of rat dACC activity result in attentional deficits (Chudasama et al, 2003; Passetti et al, 2002; Pehrson et al, 2013).

A chemogenetic approach can further these findings by providing mechanistic insights about how neuronal features mediate behavioral observations. Permanent lesions disrupt normal psychophysiology and may induce compensatory changes that confound observed cognitive alterations. Although pharmacologic approaches are temporary, they can produce off-target effects because of non-cell type-specific binding and lack of spatial control. Studies in mice facilitate chemogenetic approaches that can introduce alterations in activity with high temporal control based on specific cellular features. When coupled to behavioral paradigms, this methodology affords the opportunity to examine behavioral outcomes in conjunction with molecular-, cellular-, and circuit-level disruptions. Addressing these concerns, we expressed hM4Di in dACC neurons and subjected mice to the five-choice serial reaction time task (5CSRTT) that measures sustained visuospatial attention alongside processing speed and response control (Bari et al, 2008; Robbins, 2002). Our study was conducted in Bussey-Saksida touch-screen operant chambers with a high degree of automation and standardization, applying similar stimulus and response characteristics to those used by the analogous Cambridge Neuropsychological Test Automated Battery in humans and non-human primates (Bussey et al, 2012; Mar et al, 2013; Romberg et al, 2011; Silverman et al, 2013). By coupling DREADD technology to attentional behavior for the first time, we demonstrated that the dACC neurons play an important causal role in attention to relevant stimuli. Establishing a chemogenetic approach in a genetically tractable species opens avenues to identify specific cell-type and circuit mechanisms underlying attentional behaviors in future studies.

\section{MATERIALS AND METHODS}

\section{Animals}

Male C57Bl/6 mice (Charles River Laboratories, Massachusetts) were housed under a standard $12 \mathrm{~h}$ light/dark cycle in a temperature- and humidity-controlled vivarium. Training was initiated when mice were $10-12$ weeks old. Mice were allowed access to water for $2 \mathrm{~h}$ each day during behavioral testing. All animals were originally group-housed, but two mice (one mouse each from two cohorts subjected to behavior tests) that strongly attacked cagemates were singly housed through the duration of the experiment. Food was available ad libitum throughout the experiment. All animal protocols were approved by IACUC at Icahn School of Medicine at Mount Sinai.

\section{Drugs}

Clozapine-N-oxide (CNO; Tocris Bioscience, Bristol, UK) and pentylenetetrazole (PTZ; Sigma Aldrich, St Louis, MO) were fully dissolved in normal saline. For behavior experiments, CNO was injected intraperitoneally (i.p.) at $10 \mathrm{mg} / \mathrm{kg}$ $30 \mathrm{~min}$ before testing. For DREADD validation, mice were first separated into individual cages $1 \mathrm{~h}$ before $\mathrm{CNO}$ or vehicle injection (2-3 mice per group) to reduce variability in neuronal activation. PTZ was then administered subcutaneously $30 \mathrm{~min}$ after $\mathrm{CNO}$ injection at $65 \mathrm{mg} / \mathrm{kg}$. Mice were perfused $2 \mathrm{~h}$ later when tonic-clonic seizure was observed.

\section{Viral Injection}

Mice were anesthetized with isoflurane and head-fixed in a mouse stereotaxic apparatus (Narishige, East Meadow, NY). Bilateral injections were made at the following coordinates: $\mathrm{AP}+1.7 \mathrm{~mm}, \mathrm{ML} \pm 0.2 \mathrm{~mm}$ and $\mathrm{DV}-0.7 \mathrm{~mm}$; $\mathrm{AP}+1.1 \mathrm{~mm}$, $\mathrm{ML} \pm 0.2 \mathrm{~mm}$ and $\mathrm{DV}-0.7 \mathrm{~mm} ; \mathrm{AP}+0.4 \mathrm{~mm}, \mathrm{ML} \pm 0.2 \mathrm{~mm}$ and DV $-0.7 \mathrm{~mm}$. Then, $500 \mathrm{nl}$ of a mixture of AAV8-hSynDIO-hM4D(Gi)-mCherry and AAV8-hSyn-GFP-Cre or AAV8- CaMKIIalpha-GFP-Cre (University of North Carolina Vector Core) in a 1:1 ratio was infused in each coordinate site at $150 \mathrm{nl} / \mathrm{min}$ using a microinjector set and $2.5 \mu$ Hamilton syringe. The syringe was left in place for $1 \mathrm{~min}$ following the injection to reduce backflow of virus. We waited at least 3 weeks to allow for maximal viral expression.

\section{Five-Choice Serial Reaction Time Task}

Apparatus. Testing was conducted in the Bussey-Saksida chamber (Lafayette Instrument, Lafayette, IL), a black plastic trapezoid (walls $20 \mathrm{~cm}$ high $\times 18 \mathrm{~cm}$ wide (at screen-magazine) $\times 24 \mathrm{~cm}$ wide (at screen) $\times 6 \mathrm{~cm}$ wide (at magazine)). Stimuli were displayed on a touch-sensitive screen $(12.1$ inch, screen resolution $600 \times 800)$ divided into five response windows by a black plastic mask $\left(4.0 \times 4.0 \mathrm{~cm}^{2}\right.$, positioned centrally with windows spaced $1.0 \mathrm{~cm}$ apart, $1.5 \mathrm{~cm}$ above the floor) fitted in front of the touchscreen. Schedules were designed and data were collected and analyzed using ABET II Touch software (Lafayette Instrument). The inputs and outputs of the multiple chambers were controlled by WhiskerServer software (Lafayette Instrument).

Habituation. Before training on 5CSRTT, mice were initially trained to touch the screen. Mice were acclimated to the chambers for 3 days in 30-min sessions. During this habituation phase, the food magazine was illuminated and diluted sweetened condensed milk (Eagle Brand) was dispensed every $40 \mathrm{~s}$. Mice were then trained to touch the 
screen. A stimulus was randomly displayed in one of the five response windows. If the mouse touched the stimulus, the milk reward was delivered in conjunction with a tone and magazine light. Touches to nonstimuli had no consequence. After reaching criterion on this phase (20 touches in $30 \mathrm{~min}$ for 2 consecutive days), mice moved onto 5CSRTT training phase.

Training. Mice were tested 5 days a week, 100 trials a day (or up to $30 \mathrm{~min}$ ). Each trial began with the illumination of the magazine light. When the mouse made a nose poke in the food magazine, the stimulus was delivered after an intertrial interval (ITI) period of $5 \mathrm{~s}$. If a mouse touched the screen during this ITI period, the response was recorded as premature and the mouse was punished with a 5-s time-out (house light on). After the time-out period, the magazine light illumination and house light switch off signaled onset of the next trial. After the ITI period, a stimulus appeared randomly in one of the five response windows for a set stimulus duration (this varied from 32 to $2 \mathrm{~s}$ ). A limited-hold period followed by the stimulus duration was $5 \mathrm{~s}$, during which the stimulus was absent but the mouse was still able to respond to the location. Responses during stimulus presence and limited holding period could be recorded either as correct (touching the stimulus window) or incorrect (touching any other windows). A correct response was rewarded with a tone, and milk delivery, indicated by the illumination of the magazine light. A failure to respond to any window over the stimulus and limited-hold period was counted as an omission. Incorrect responses and omissions were punished with a 5-s time-out. In addition, animals could make perseverative responses that are screen touches after a correct or incorrect response. Animals started at a stimulus duration of $32 \mathrm{~s}$. With a goal to baseline mice at a stimulus duration of $2 \mathrm{~s}$, the stimulus duration was sequentially reduced from 32,16 , 8,4 , to $2 \mathrm{~s}$. Animals had to reach a criterion ( $>50$ trials, $>80 \%$ accuracy, $<20 \%$ omissions) over 2 consecutive days to pass from one stage to the next. After reaching baseline criterion with the $2 \mathrm{~s}$ stimulus duration (5 consecutive days), mice were injected with virus.

Reduced stimulus test. Following viral injections, mice were reestablished to baseline and then challenged with an increased attentional demand by reducing the stimulus duration to 2, 1.5, 1, and $0.8 \mathrm{~s}$ (reduced stimulus test). They then underwent 8 days of testing, with half of the mice receiving vehicle injections on odd days and the other half on even days. After 4 days of testing, mice were subjected to $2 \mathrm{~s}$ stimulus duration training to confirm that the mice maintain stable baseline performance. In case of under baseline criteria, the mice were reestablished to baseline, and then were tested. Attention and response control were assessed by measuring the following performance: percentage accuracy $(100 \times$ correct responses/(correct responses +incorrect responses)), percentage omission $(100 \times$ omissions/(omissions+correct responses+incorrect responses)), number of premature responses, number of perseverative responses, latency to collect response, and latency to reward collection after correct choices.

\section{Open Field Test}

Locomotor activity was measured for $30 \mathrm{~min}$ in a square apparatus $(43 \mathrm{~cm} \times 43 \mathrm{~cm} \times 33 \mathrm{~cm})$ equipped with a panel of infrared beams ( 16 beams) located in the horizontal direction along the sides of each square apparatus. Data were collected with Fusion v4 software (Omnitech Electronics, Columbus, $\mathrm{OH})$. Mice were subjected to the open field test more than 1 week after the completion of 5CSRTT. Activity was assessed twice for each mouse (with vehicle and CNO injection respectively). The order of vehicle versus CNO administration was counterbalanced across mice. Activity sessions were separated by at least 1 week drug-free days.

\section{Immunohistochemistry}

Anesthetized mice were transcardially perfused with cold 4\% paraformaldehyde (PFA) dissolved in $0.1 \mathrm{M}$ phosphate buffer. The brains were post-fixed in $4 \%$ PFA at $4{ }^{\circ} \mathrm{C}$, and cryoprotected in $30 \%$ sucrose solution. The frozen brains were sectioned into 40 - $\mu \mathrm{m}$-thick coronal sections using a cryostat (CM3050, Leica). Free-floating sections were washed in tris-buffered saline (TBS), $\mathrm{pH}$ 7.5, and then blocked in $1 \%$ bovine serum albumin in TBST $(0.25 \%$ Triton $\mathrm{X}-100$ in TBS) for $1 \mathrm{~h}$. The sections were incubated with antic-Fos antibody (1:500; sc-52, Santa Cruz Biotechnology) or anti-CamKII antibody ( $1: 200 ; 05-532$, EMD Millipore) overnight at room temperature. They were washed in TBST, followed by incubation with Alexa 647-conjugated goat antirabbit antibody or Alexa 647-conjugated donkey anti-mouse antibody (Thermo Fisher Scientific). To visualize all neurons, we used NeuroTrace 435/455 blue fluorescent Nissl stain (Thermo Fisher Scientific). This fluorescent probe has been reported to only label neurons as stained cells do not colocalize with markers of glial cells, particularly astrocytes, microglia, or oligodendrocytes (Lazo et al, 2010).

\section{Double Fluorescence In Situ Hybridization}

In situ hybridization was performed as described previously (Demars and Morishita, 2014). cDNA fragments of mouse vGlut1 and Cre were amplified by PCR. Probes were synthesized using T3/T7 RNA polymerase (Roche) labeled with digoxigenin or fluorescein and hybridized to frozen sections. After overnight hybridization of RNA probes at $72{ }^{\circ} \mathrm{C}$, sections were incubated with anti-fluorescein POD antibodies $(1: 2000)$ followed by TSA Plus DNP signal amplification (Perkin Elmer), and then incubated with anti-DIG-alkaline phosphatase and anti-DNP-KLH-488 (both $1: 1000$, Roche). Following incubation, slides were incubated in fast red solution (Roche). Finally, slides; were washed in water and coverslipped using CC mount solution (Sigma Aldrich). For colocalization counting, four images/ mouse from two mice were taken around injection sites.

\section{Imaging and Quantification}

Imaging was performed using LSM780 confocal microscopes (Zeiss). All images were processed and analyzed on ImageJ software $(\mathrm{NIH})$. Images were thresholded using the Max Entropy algorithm. Total cell numbers were estimated using the 'Analyze Particle' function. For analysis of colocalization 
a
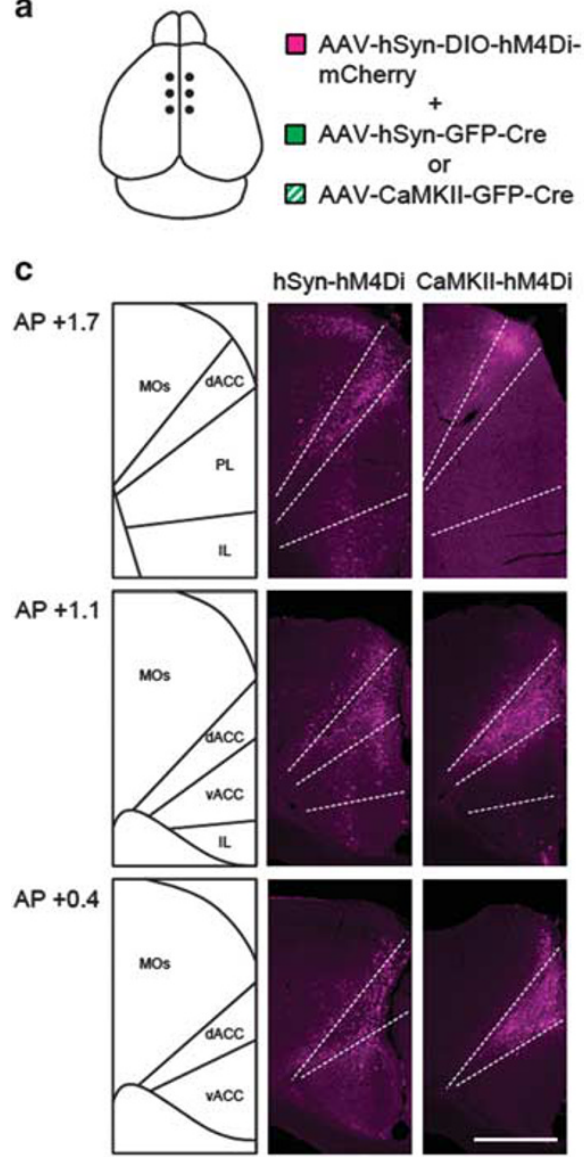

b GFP-Cre/hM4Di-mCherry hSyn-hM4Di CaMKII-hM4Di
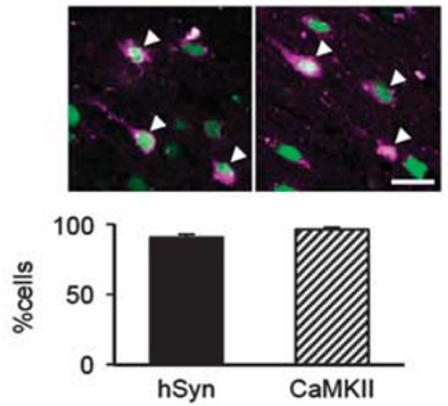

d

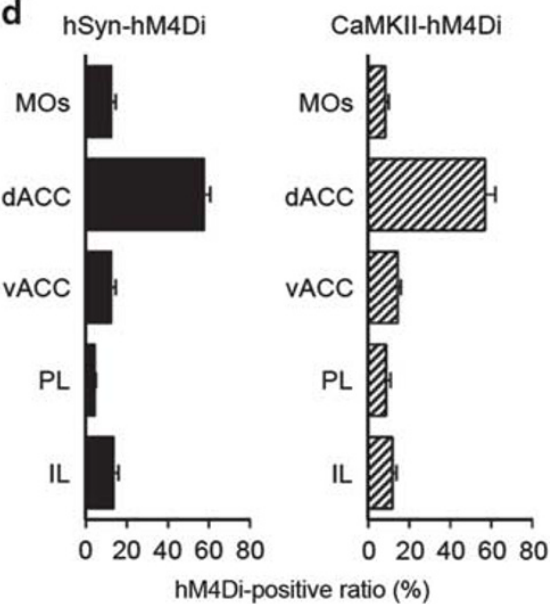

Figure I Viral transduction of hM4Di in the dorsal anterior cingulate cortex neurons. (a) Experimental approach for Cre-dependent inhibitory DREADD showing stereotactic injection coordinates. (b, top panel) Representative confocal images showing native fluorescent signals from dACC cells coexpressing hM4Di-mCherry (magenta) and GFP-Cre (green). GFP-Cre was transduced with the hSyn promotor (left panel) or CaMKllo promotor (right panel). Arrowheads indicate cells coexpressing hM4Di and Cre (white). Scale bar $=20 \mu \mathrm{m}$. (b, bottom panel) Quantification of the percentage of hM4Di-positive cells that coexpress Cre. Data are represented as mean \pm SEM $(n=6)$. (c) Representative confocal images showing native hM4Di-mCherry fluorescence (magenta) in the prefrontal cortex of hSyn-hM4Di and CaMKII-hM4Di mice. MOs, secondary motor; dACC, dorsal anterior cingulate; vACC, ventral anterior cingulate; PL, prelimbic; IL, infralimbic. Scale bar $=500 \mu \mathrm{m}$. (d) Relative distribution of hM4Di-positive cells transduced by AAV-hSyn-GFP-Cre (left panel) or AAVCaMKIll $\alpha$-GFP-Cre virus (right panel) among prefrontal cortices. Data are represented as mean \pm SEM $(n=6)$.

with Nissl or GFP-Cre, the analysis was performed with three sections/mouse from six mice for each group. For CaMKII immunohistochemistry, 8-10 images/each mouse were taken from 2 hSyn mice and 2 CaMKII mice. For hM4Di distribution, to ensure the coverage of the anterior-posterior axis for our counting, three sections per hemisphere were collected from three different areas around each injection site (one section each from each injection site) spanning anterior-posterior axis $(\mathrm{AP}+1.7,+1.1,+0.4 \mathrm{~mm})$. Six images were collected from each animal, and were averaged from six mice for the quantification. Although our quantification does not include the most anterior $(\mathrm{AP}+2.2 \mathrm{~mm})$ and posterior $(\mathrm{AP}-0.1 \mathrm{~mm})$ portion of $\mathrm{ACC}$, we expect the reported widespread transduction of AAV8 (Aschauer et al, 2013) allows the viral transduction to extend up to $500 \mu \mathrm{m}$ from the injection sites close to the anterior and posterior boarder of ACC. Nonetheless, we cannot rule out the possibility that our hM4Di distribution analysis overestimated the transduction rate. For c-Fos/hM4Di colocalization analysis, 4-6 images/mouse were taken around injection sites (vehicle- and PTZ-treated hSyn-hM4Di mice, $n=3$; CNO- and PTZ-treated hSyn-hM4Di mice, $n=3$; vehicle- and
PTZ-treated CaMKII-hM4Di mice, $n=2$; CNO- and PTZ-treated CaMKII-hM4Di mice, $n=3$ ).

\section{Statistical Analyses}

Statistical analyses were performed using Prism (Graphpad). The 5CSRTT data (accuracy and omission) were analyzed using a two-way repeated measures analysis of variance (ANOVA) with Drug (vehicle, CNO) and Stimulus duration $(2,1.5,1,0.8 \mathrm{~s})$ as within-subjects factors, followed by post hoc Bonferroni's multiple comparisons test when F-ratios of the interaction were significant $(p<0.05)$. In the analysis of other 5CSRTT data and locomotion, a paired $t$-test was used. Immunohistochemistry data were analyzed using Student's $t$-test. All data are expressed as means \pm SEM.

\section{RESULTS}

\section{Targeting Inhibitory DREADD to dACC Neurons}

To achieve cell type-specific suppression of dACC neuronal activation in mice, we bilaterally injected 
AAV8-hSyn-DIO-hM4Di-mCherry with AAV8-hSyn-GFPCre (hSyn-hM4Di mice) for pan-neuronal transduction or AAV8-CaMKII $\alpha$-GFP-Cre (CaMKII-hM4Di mice) for excitatory neuron-specific transduction (Figure 1a). hM4Di was expressed in a Cre-dependent manner: $91 \pm 2 \%$ and $96 \pm 1 \%$ of hM4Di-positive cells coexpressed Cre in hSynhM4Di and CaMKII-hM4Di mice, respectively (Figure 1b). hM4Di transduction was neuron specific: $95 \pm 2 \%$ and $97 \pm 1 \%$ hM4Di-positive cells were costained with Nissl in hSyn-M4Di and CaMKII-hM4Di mice respectively (Supplementary Figure S1a and b). We also confirmed that AAV-CaMKII $\alpha$-GFP-Cre was selectively expressed in excitatory neurons: $94 \pm 2 \%$ of hM4Di-positive cells were immunolabeled with an excitatory neuron marker CaMKII protein in CaMKII mice, whereas $86 \pm 4 \%$ of hM4Di-positive cells coexpressed CaMKII in hSyn mice consistent with the promotor selectivity (Supplementary Figure S1c and d). Double in situ hybridization of Cre with vGlut1, a marker of glutamatergic neurons (Supplementary Figure S1e and f), also showed $89 \pm 4 \%$ of the total Cre-positive cells in the dACC coexpressed vGlut1 mRNA in CaMKII mice, demonstrating that AAV-CaMKII $\alpha$-GFP-Cre transfection was highly selective toward excitatory neurons. In addition, $17 \pm 3 \%$ and $26 \pm 4 \%$ of Nissl-positive dACC neurons expressed hM4Di in hSyn-hM4Di and CaMKII-hM4Di mice, respectively (data not shown). We thus found that hM4Di expressed in a pan-neuronal manner in hSyn-hM4Di mice and in excitatory neurons in CaMKII-hM4Di mice.

To assess specificity of transduction to the dACC, we mapped the pattern of hM4Di expression in the prefrontal cortex (Figure 1c and d). The largest percentage of hM4Di-expressing cells was in the dACC in hSyn-hM4Di $(58 \pm 3 \%)$ and CaMKII-hM4Di mice $(57 \pm 5 \%)$. Expression ratios of hM4Di in secondary motor (MOs), ventral ACC (vACC), prelimbic (PL), and infralimbic (IL) cortices in hSyn-hM4Di mice were $12 \pm 2 \%, 12 \pm 2 \%, 4 \pm 1 \%$, and $14 \pm 2 \%$, respectively. Expression ratios of CaMK2-hM4Di in MOs, vACC, PL, and IL were $8 \pm 2 \%, 14 \pm 2 \%, 9 \pm 2 \%$, and $12 \pm 2 \%$, respectively. Our quantification shows that hM4Di was predominantly expressed in the dACC in both hSyn- and CaMKII-hM4Di mice.

Although the inhibitory DREADD virus that we used has been reported to suppress firing of infected neurons (Perova et al, 2015), we additionally verified its functional effect in vivo by assaying expression of c-fos, a recognized marker of neuronal activation, upon $\mathrm{CNO}$ administration. As previous reports suggest that high baseline c-fos activation is required to detect the reduced c-fos activation by $\mathrm{CNO}$ (Koch et al, 2015; Sasaki et al, 2011), we elevated the baseline c-fos activation with PTZ $(65 \mathrm{mg} / \mathrm{kg})$, a $\mathrm{GABA}_{\mathrm{A}}$ receptor antagonist (Andre et al, 1998; Li et al, 2014), to allow for in vivo quantification. We found that CNO $(10 \mathrm{mg} / \mathrm{kg})$ significantly decreased the percentage of total hM4Diexpressed cells that coexpress c-fos in both hSyn- and CaMKII-hM4Di mice injected with PTZ (Supplementary Figure S2). Furthermore, c-Fos immunohistochemistry in mice perfused $60 \mathrm{~min}$ after the reduced stimulus 5CSRTT showed that CNO slightly, but not significantly, suppressed the reduced stimulus test-induced c-Fos expression (\% of c-Fos+ cells in hM4Di+ cells: $13 \pm 3 \%$ with CNO vs $18 \pm 9 \%$ with saline in hSyn-hM4Di mice. CNO or vehicle was given
30 min before the test; 3 mice each). Based on prior reports (Koch et al, 2015; Sasaki et al, 2011), we concluded that this finding is likely because of the far lower baseline c-fos activation of ACC neurons by attention task compared with PTZ-induced c-Fos activation $(18 \pm 9 \%+5$ CSRTT vs $29 \pm 3 \%+$ PTZ: Supplementary Figure S2). Although hM4Di suppression of PTZ-induced c-Fos expression does not directly prove that $\mathrm{CNO}$ suppresses attention-dependent neural activity, it does however provide a proof of principle that CNO-dependent inactivation of neural activity in cells expressing inhibitory DREADD applies to viral introduction to the dACC in vivo.

\section{Reversible Chemogenetic Inactivation of the dACC Neurons Disrupts Attentional Behavior}

Once we established that inhibitory DREADD was functioning to suppress dACC neuronal activity, we subjected mice to the 5CSRTT. After training mice to baseline (see the detailed method in Materials and Methods section), AAVs were injected as shown in Figure 1. Mice were maintained at a performance baseline for 3 weeks in order to achieve maximal hM4Di expression before testing. During the test phase, $\mathrm{CNO}$ or saline was administered at $10 \mathrm{mg} / \mathrm{kg} 30 \mathrm{~min}$ before each of test sessions. The injection schedule was counterbalanced across mice (Figure 2a). Because pharmacokinetic studies report that CNO given i. p. at $1 \mathrm{mg} / \mathrm{kg}$ is eliminated from plasma within $2 \mathrm{~h}$ and reaches peak efficacy for $10 \mathrm{~min}$ to $10 \mathrm{~h}$, we waited $30 \mathrm{~min}$ until testing and allowed $24 \mathrm{~h}$ for the drug effect to dissipate fully (Farrell and Roth, 2013; Ray et al, 2011). In the absence of DREADD expression, CNO administration at dose of $\geq 10 \mathrm{mg} / \mathrm{kg}$ has been reported to have no measurable effect across many monitored behavioral and physiological assays (Alexander et al, 2009; Mahler et al, 2014; Ray et al, 2011; Richards et al, 2014).

In each test session, mouse attention demand was taxed by reducing the stimulus duration to $2,1.5,1$, and $0.8 \mathrm{~s}$ in a pseudorandomized order. Prior studies have shown that attention deficits can be measured quantitatively as a decrease in accurate responses and an increase in omitted responses to light stimulus, and that performance decreases with shortened stimulus duration. Consistent with previous findings, as the stimulus duration decreased, all mice showed an expected increase in the percentage of omission and decrease in the percentage of accuracy (omission: hSynhM4Di mice: $\mathrm{F}(3,15)=22.06, p<0.001$; CaMKII-hM4Di mice: $\mathrm{F}(3,15)=11.52, \quad p<0.001$; accuracy: hSyn-hM4Di mice: $\mathrm{F}(3,15)=24.40, \quad p<0.001$, CaMKII-hM4Di mice: $\mathrm{F}(3,15)=29.86, p<0.001$ ) (Figure $2 \mathrm{~b}-\mathrm{e}$ ). We observed a difference between hSyn-hM4Di mice and CaMKII-hM4Di mice in the omission rate at $2.0 \mathrm{~s}$ when treated with vehicle, but we saw no difference in the omission rate between mice injected with DREADD and controls injected with GFP (H Koike and H Morishita, unpublished observation: GFP vs hSyn DREADD ( $p=0.40, t$-test), GFP $v s$ CaMKII DREADD $(p=0.17, t$-test $))$. We therefore consider this difference to represent normal performance variability across the population of injected mice subjected to the 5CSRTT.

We next assessed accurate and omitted trials as stimulus duration decreased in both groups upon CNO administration (Bari et al, 2008; Mar et al, 2013). Compared with 
a

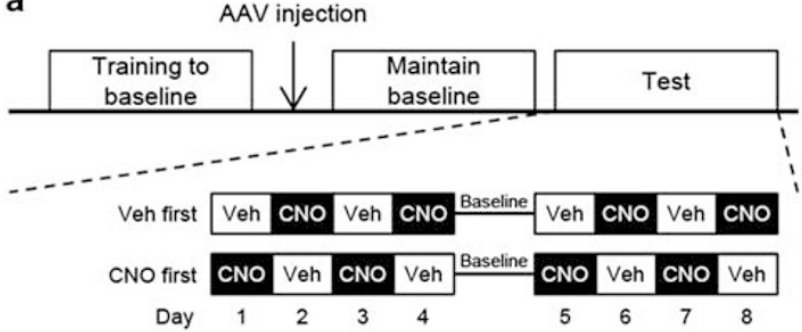

b

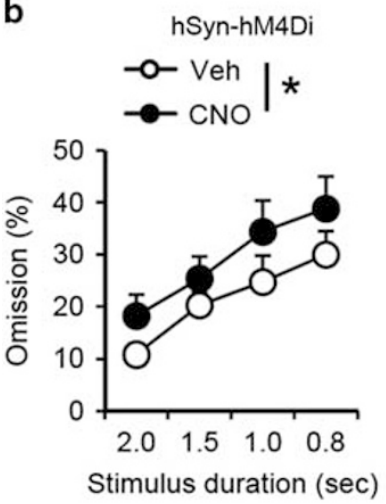

C

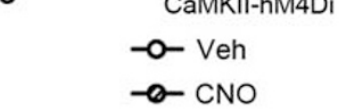

d

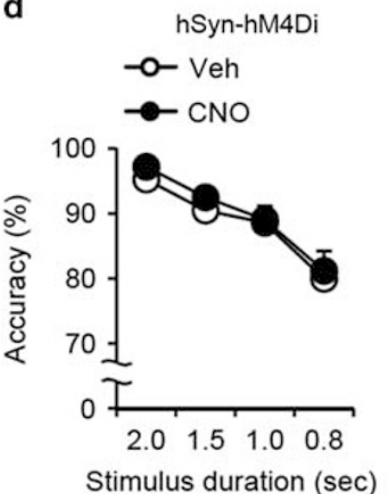

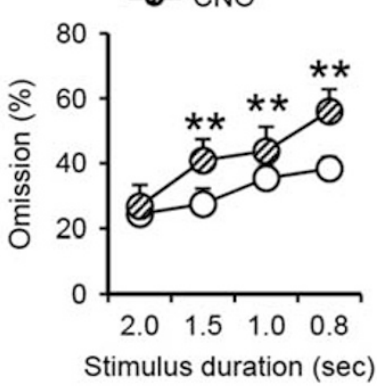

e

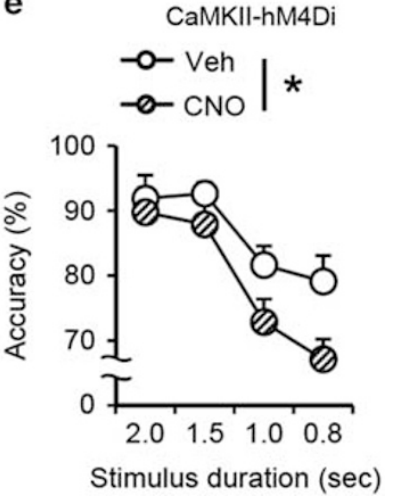

Figure 2 DREADD inactivation of $\mathrm{dACC}$ neurons produces visual attention deficits. (a) Schematic overview of the experimental design. All mice were injected with $A A V s$ after reaching baseline criteria. Baseline performance was maintained whereas hM4Di expression reached full expression. Animals were divided into counterbalanced groups that received either vehicle or $\mathrm{CNO}$ on the first day of testing; both groups alternated between $\mathrm{CNO}$ and vehicle for each testing session. Injections were administered $30 \mathrm{~min}$ before testing and one testing session was executed each day. (b, c) CNO administration increases the percentage of trials omitted. (d, e) CNO administration does not affect accuracy when hM4Di was expressed pan-neuronally, but reduces the percentage of accurate responses when expressed in excitatory neurons. Veh, vehicle; CNO, clozapine-N-oxidate. ${ }^{*} * P<0.01$, ${ }^{*} p<0.05$ vs vehicle. Data are represented as mean $\pm \operatorname{SEM}(n=6)$.

vehicle delivery, $\mathrm{CNO}$ administration significantly increased the number of trials omitted in both hSyn-hM4Di and CaMKII-hM4Di mice. In hSyn-M4Di mice we observed a significant drug effect across all stimulus durations (main effect of drug: $\mathrm{F}(1,5)=8.743, p<0.05$; interaction between drug and stimulus duration: $\mathrm{F}(3,15)=0.40, p=0.76)$ and in CaMKII-hM4Di mice the drug effect gained significance as stimulus duration shortened below baseline (main effect of drug: $\mathrm{F}(1,5)=3.915, p=0.10$; interaction: $\mathrm{F}(3,15)=11.21$,

$p<0.001$; post hoc comparison for $1.5,1.0,0.8 \mathrm{~s} p<0.01$ ) (Figure $2 \mathrm{~b}$ and $\mathrm{c}$ ). $\mathrm{CNO}$ administration similarly significantly decreased accuracy in CaMK2-hM4Di mice but not in hSyn-hM4Di mice (CaMK2-hM4Di mice: main effect of drug: $\mathrm{F}(1,5)=10.52, p<0.05$; interaction: $\mathrm{F}(3,15)=0.87$, $p=0.48$; hSyn-hM4Di mice: main effect of drug: $(\mathrm{F}(3,15)=0.99, \quad p=0.37 ; \quad$ interaction: $\mathrm{F}(3,15)=0.11$, $p=0.95$ ) (Figure 2d and e). Of note, 5CSRTT performances at $0.8 \mathrm{~s}$ were not changed throughout testing (hSyn-hM4Di: effect of day for omission: $\mathrm{F}(3,36)=2.14, p=0.11$; effect of day for accuracy: $\mathrm{F}(3,36)=0.21, p=0.88$; CaMKII-hM4Di mice: effect of day for omission: $\mathrm{F}(3,30)=2.54, p=0.07$; effect of day for accuracy: $F(3,30)=1.32, \quad p=0.28)$ (Supplementary Figure S3), indicating repeating tests and CNO treatment did not influence each other's tests. Collectively, these results suggest that chemogenetic inactivation of the dACC neurons disrupts attention behavior.

\section{Reversible Chemogenetic Inactivation of dACC Neurons Did Not Cause Deficits in Motivation and Locomotion}

Because an increase in omission can also arise because of altered motor function and decreased motivation to obtain a reward, we next sought to evaluate motivation and motor deficits through latency to collect rewards as well as the open field test (Bari et al, 2008; Mar et al, 2013). Although we observed a significant lengthening of latency to produce a correct response in hSyn-hM4Di mice $(p<0.05)$, but not in CaMK2-hM4Di mice $(p=0.13)$, CNO administration did not affect latency to collect reward (hSyn-hM4Di, $p=0.40$; CaMKII-hM4Di, $p=0.13$ ) (Figure $3 \mathrm{a}-\mathrm{d}$ ). CNO administration did not affect the distance moved in the open field test (hSynhM4Di, $p=0.88$; CaMKII-hM4Di, $p=0.29$ Figure $3 e$ and f), ruling out motor deficits. Collectively, these results demonstrate that increased omission and processing speed deficit were likely not induced secondarily by lack of motivation or gross motor deficits.

\section{Reversible Chemogenetic Inactivation of dACC Neurons Did Not Induce Impulsive and Compulsive Behavior}

To examine whether the effect of chemogenetic inactivation of dACC neurons is specific to attention processing, we also assessed for alterations in impulsivity and compulsivity upon $\mathrm{CNO}$ administration by measuring the number of premature responses and perseverative responses, respectively (Bari et al, 2008; Mar et al, 2013). No effect of CNO administration on the number of premature responses (Figure 4a and b: hSyn-hM4Di, $p=0.71$; CaMKII-hM4Di, $p=0.50$ ) and perseverative responses (Figure $4 \mathrm{c}$ and d: hSynhM4Di, $p=0.15$; CaMKII-hM4Di, $p=0.30$ ) was observed in either hSyn-hM4Di or CaMKII-hM4Di mice, indicating that response control was not affected by the dACC pan-neuronal or excitatory inactivation.

\section{DISCUSSION}

By combining a chemogenetic neural silencing approach with a translational, touchscreen-based attention task in mice, we demonstrated that DREADD inactivation of the dACC neurons disrupts attentional behavior. Our chemogenetic approach circumvents the difficulties in interpretations 
a

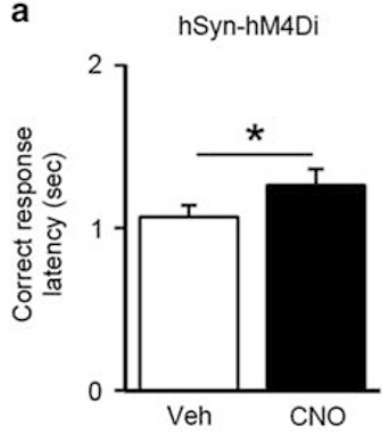

c

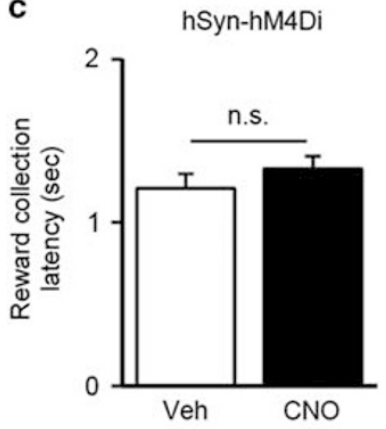

e

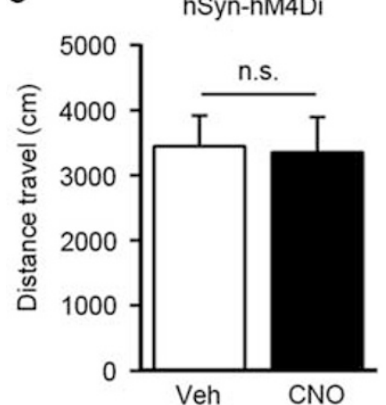

b

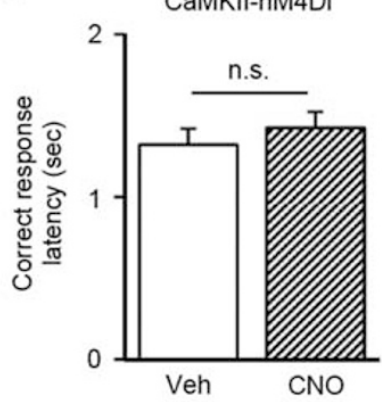

d

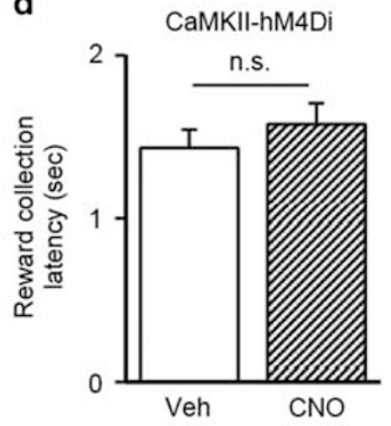

f

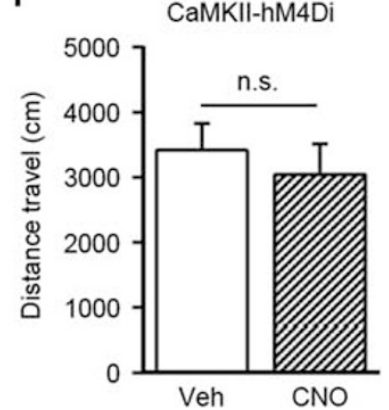

Figure 3 DREADD inactivation of dACC neurons does not affect processing speed, motivation, or locomotion. (a, b) In hSyn-hM4Di but not CaMKII-hM4Di mice, CNO administration increased collect response latency. (c, d) CNO administration does not affect the latency to collect reward for a correct response collection latency. (e, f) In a separate open field test, the average distance traveled is not altered by DREADD inhibition. Veh, vehicle; CNO, clozapine-N-oxidate. ${ }^{* P}<0.05$ vs vehicle; NS, nonsignificant. Data are represented as mean $\pm \operatorname{SEM}(n=6)$.

associated with the lesion or pharmacological studies by directly establishing a causal role of dACC neural activities on attentional behavior. Our study supports the possibility that the changes in dACC observed in many psychiatric disorders (Bush et al, 1999; Goodkind et al, 2015) can causally contribute to cognitive dysfunction. As far as we are aware, this is the first study that directly demonstrates that dACC neurons play a causal role in attention processing in mice. Compared with optogenetic techniques, chemogenetic approaches are less invasive and permit neural activity manipulations over extended periods of time; this approach is thus ideally suited to examine the long-term impacts of sustained altered neural activity over the time course of developmental time windows. Such manipulations will enable study of how normal developmental contributions

a

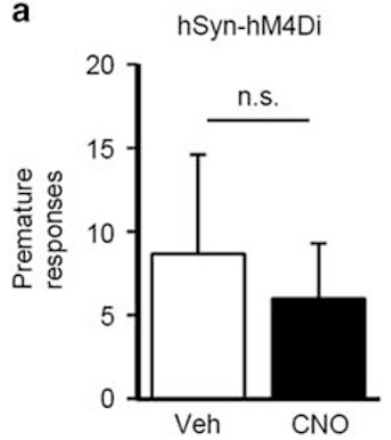

b
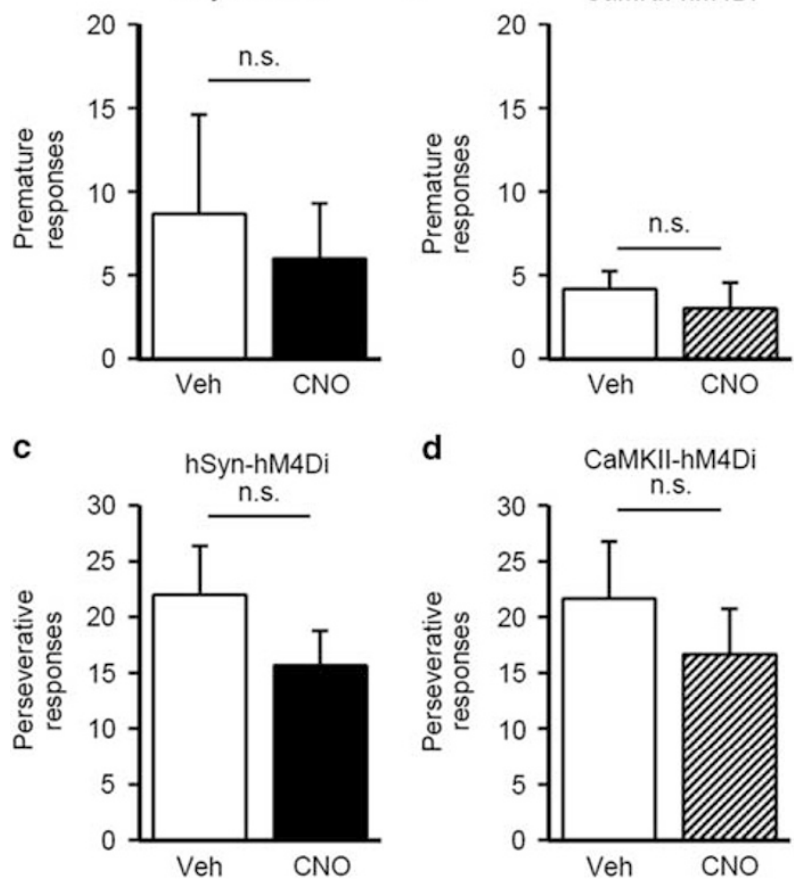

d

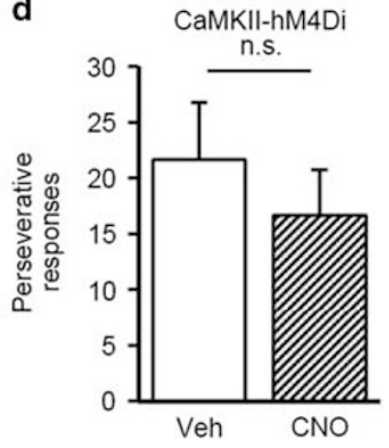

Figure 4 DREADD inactivation of $d A C C$ neurons does not alter impulsivity and compulsivity. (a, b) The number of premature responses before the display of the light stimulus does not change under the influence of CNO. (c, d) Repetitive nose-poking in response to the light stimulus is not affected by drug administration. Veh, vehicle; $\mathrm{CNO}$, clozapine- $\mathrm{N}$ oxidate; NS, nonsignificant. Data are represented as mean \pm SEM $(n=6)$.

produce long-lasting impacts on attention, and additionally, how aberrant developmental signaling could be targeted to prevent attentional deficits in preclinical models. DREADD can be expressed in cell-type and circuit-specific manners via viral approaches (Urban and Roth, 2015). Because of this capability, our study provides a template for future studies to chemogenetically dissect the neural circuit basis of attentional behavior in mice.

We observed that dACC neuronal inactivation by DREADD decreases accuracy and increases omissions in mice during attention behavior assayed with the 5CSRTT (Figure 2). Although omissions can indicate an attention deficit as mice fail to detect the presented stimulus, it is also altered when mice have motor deficits that prevent them from responding in a timely manner, or lack motivation to obtain a reward received following a correct response (Robbins, 2002). Given that latency to reward and locomotor activity were not affected by the DREADD inactivation (Figure 3), it is highly likely that the observed increase in omissions by dACC inhibitory DREADD primarily reflects a deficit specific to attention. Minor inactivation of $\sim 20 \%$ of the dACC neuronal population could induce attention deficits, suggesting that the dACC provides prominent contributions to normal attention-based cognition. The specificity of our DREADD inactivation also points to a specific role for the activity of dACC neurons in attentionbased behavior, and not in impulsivity and compulsivity (Figure 4). Our results are consistent with a previously 
reported lesion study in rat dACC (Chudasama et al, 2003), but more conclusively demonstrated that dACC neuronal activity is a key contributor to attention-based cognition without the confounding nonspecific lesions that accompany lesions.

Our pan-neuronal and excitatory inactivation produced overlapping yet separate effects on attention processing (Figure 2), suggesting that more elaborate cell type-specific inactivation will be important in future studies to draw conclusions about the neural mechanisms underlying this type of cognition. Pan-neuronal dACC inactivation induced mild attentional deficits and slowed processing speed. In contrast, excitatory neuron-specific inactivation produced more severe effects on attention with no impact on processing speed. Because transduction efficacy was comparable between hSyn-hM4Di and CaMKII-hM4Di mice (Figure 1: $p=0.11$, paired $t$-test), the different attention deficits between the two groups appear to reflect differences in behavioral contributions from infected cell types. The accuracy deficit we observed in the inhibition of excitatory neurons may be masked by pan-neuronal inactivation: inhibitory neuron inactivation often produces excitatory cell disinhibition, and this effect may in fact compensate for the primary action of DREADD inhibition on excitatory neurons. To our surprise, pan-neuronal inactivation, compared with excitatory neuron-specific inactivation, produced a greater processing speed deficit with a milder attention deficit. Interestingly, a similar phenotype (increased correct response latency without accuracy deficits in the 5CSRTT) was previously reported following microinjection in rat PFC of a NMDA receptor antagonist that preferentially binds and inhibits GABAergic neural activity and enhances glutamatergic neural activity (Carli et al, 2006; Homayoun and Moghaddam, 2007). As hM4Di likely inactivates nonexcitatory GABAergic neurons in hSyn mice, but not in CaMKII mice (Supplementary Figure $1 \mathrm{c}$ and $\mathrm{d}$ ), we speculate that GABAergic neuron inactivation could contribute to the increased correct response latency in hSyn mice. Furthermore, an activity balance between glutamatergic and GABAergic neurons could differentially contribute to the dissociation between the processing speed and attention phenotypes in hSyn and CaMKII mice. Taken together, our findings suggest that activity balances of excitatory and inhibitory neurons in the dACC play a critical role in attentional processes and processing speed (Pehrson et al, 2013; Totah et al, 2009). More elaborated cell type-specific inactivation by specific gene promoters in excitatory and inhibitory neurons could shed light on the neural mechanisms of attention and processing speed in the dACC in future studies.

The dACC is connected with many different brain regions and attention processing likely requires signaling in additional areas. Interestingly, the dACC is reciprocally connected with visual area, and 'top-down' dACC projections modulate sensory processing in the visual cortex (Zhang et al, 2014; Zingg et al, 2014). Thus, one possibility is that attention deficits observed in our study could be attributed to dACC inactivation or alteration of top-down visual processing. Another potential explanation is that dACC inactivation disrupts the passage of visual information based on the stimulus to connected somatomotor areas that mediate responsive motor activity (Zingg et al, 2014). It is also possible that a functional interaction between ACC and PL neurons, associated with preparatory attention in rats, could be disrupted in the present study (Totah et al, 2013). Our Cre-dependent viral approach in mouse provides a template for future circuit-specific chemogenetic studies for alternative combinatorial approaches to address such possibilities; replacing AAV8-Cre with retrograde traveling Canine adenovirus type 2 (CAV)-Cre in axonal projecting areas will permit Cre expression only within unique projection circuits (Boender et al, 2014; Carter et al, 2013; Penzo et al, 2015). This chemogenetic dissection approach can serve as a powerful tool to deduce which circuits inhibited by global dACC inactivation primarily contribute to attention deficits. Finally, noninvasive nature of chemogenetic approach is particularly well-suited to examine how established dynamic changes in network activity across adolescence contribute to brain development (Booth et al, 2003; Fair et al, 2007).

Although our study proposes a new neurobiological basis underlying 5CSRTT performance in mice, it would be fruitful to extend a chemogenetic approach to other attention tasks in future studies. Although 5CSRTT is the most widely used task to study the neural basis of attention in rodents (Lustig et al, 2013; Moore et al, 2013), there are some limitations (Young et al, 2012; Young and Geyer, 2015). First, 5CSRTT does not to contain nontarget stimuli requiring subjects to inhibit their responses that are included in the continuous performance task (CPT), a gold standard of attention task in human patients. A variant of the 5CSRTT, the rodent five-choice continuous performance test (5C-CPT), requires response inhibition to irrelevant stimuli and provides greater consistency with the human CPT (Young et al, 2009). Second, the primary measure of attention deficits in 5CSRTT performance is inconsistent between humans and rodents (Young et al, 2013b). In the human 5CSRTT, schizophrenia patients show increased variability in reaction times with no accuracy and omission deficits (Barnett et al, 2010); however, attention performance in rodent 5CSRTT is assessed mainly by accuracy and omission. As the 5C-CPT, similar to the human CPT, permits performance analysis using signal detection theory, it may have a better translational validity for the human CPT (Young et al, 2013a). Applying the DREADD technology we used here to $5 \mathrm{C}$-CPT would greatly facilitate the translation of findings in animal studies for potential treatments for humans. Finally, the National Institute of Mental Health Research Domain Criteria (RDoC) project recommends a divided attention task as a primary measurement of attention in humans (Cuthbert, 2015; Insel et al, 2010). Future efforts to establish a well-designed divided attention task (target selection in the presence of competition) (Arnold et al, 2003) together with chemogenetic manipulation would be another important direction.

In the present study, we demonstrated a causal relationship between the dACC neuronal activity and visual attention, and established a foundation for applying a chemogenetic approach in genetically tractable species. Combining chemogenetic technology with translational behavior tasks in mouse will be a useful strategy to understand the neural basis of attention in more detail at cell-type and circuit levels to reveal how attentional deficits are caused in psychiatric disorders, and also enable the more 
precise delineation of drug effects on attention deficits by identifying the mechanism of treatment-induced improvement of attentional control in preclinical animal models.

\section{FUNDING AND DISCLOSURE}

This work was supported by 5T32DA007135-29 (to MPD), P50MH096890 (to SA), March of Dimes (to HM), Whitehall Foundation (to HM), Brain and Behavior Research Foundation (to HM), and NIH (to SA and MGB). HK is an employee of Taisho Pharmaceutical. The authors declare no conflict of interest.

\section{ACKNOWLEDGMENTS}

We thank Ms Tess Veuthey for assistance with touchscreen behavior system, Dr Yasmin Hurd and Dr Michael Michaelides for their expertise on DREADD, and Dr Yang Jiang for assisting open field tests.

\section{REFERENCES}

Alexander GM, Rogan SC, Abbas AI, Armbruster BN, Pei Y, Allen JA et al (2009). Remote control of neuronal activity in transgenic mice expressing evolved $\mathrm{G}$ protein-coupled receptors. Neuron 63: 27-39.

Alexander MP, Stuss DT, Shallice T, Picton TW, Gillingham S (2005). Impaired concentration due to frontal lobe damage from two distinct lesion sites. Neurology 65: 572-579.

Andre V, Pineau N, Motte JE, Marescaux C, Nehlig A (1998). Mapping of neuronal networks underlying generalized seizures induced by increasing doses of pentylenetetrazol in the immature and adult rat: a c-Fos immunohistochemical study. Eur J Neurosci 10: 2094-2106.

Arnold HM, Bruno JP, Sarter M (2003). Assessment of sustained and divided attention in rats. Curr Protoc Neurosci Chapter 8: Unit 8.5E.

Aschauer DF, Kreuz S, Rumpel S (2013). Analysis of transduction efficiency, tropism and axonal transport of AAV serotypes 1, 2, 5, 6, 8 and 9 in the mouse brain. PLoS One 8: e76310.

Bari A, Dalley JW, Robbins TW (2008). The application of the 5-choice serial reaction time task for the assessment of visual attentional processes and impulse control in rats. Nat Protoc 3: 759-767.

Barnett JH, Robbins TW, Leeson VC, Sahakian BJ, Joyce EM, Blackwell AD (2010). Assessing cognitive function in clinical trials of schizophrenia. Neurosci Biobehav Rev 34: 1161-1177.

Boender AJ, de Jong JW, Boekhoudt L, Luijendijk MC, van der Plasse G, Adan RA (2014). Combined use of the canine adenovirus-2 and DREADD-technology to activate specific neural pathways in vivo. PLoS One 9: e95392.

Booth JR, Burman DD, Meyer JR, Lei Z, Trommer BL, Davenport ND et al (2003). Neural development of selective attention and response inhibition. Neuroimage 20: 737-751.

Brown RG, Scott LC, Bench CJ, Dolan RJ (1994). Cognitive function in depression: its relationship to the presence and severity of intellectual decline. Psychol Med 24: 829-847.

Bush G, Frazier JA, Rauch SL, Seidman LJ, Whalen PJ, Jenike MA et al (1999). Anterior cingulate cortex dysfunction in attentiondeficit/hyperactivity disorder revealed by fMRI and the Counting Stroop. Biol Psychiatry 45: 1542-1552.

Bussey TJ, Holmes A, Lyon L, Mar AC, McAllister KA, Nithianantharajah J et al (2012). New translational assays for preclinical modelling of cognition in schizophrenia: the touchscreen testing method for mice and rats. Neuropharmacology 62: 1191-1203.

Carli M, Baviera M, Invernizzi RW, Balducci C (2006). Dissociable contribution of 5-HT1A and 5-HT2A receptors in the medial prefrontal cortex to different aspects of executive control such as impulsivity and compulsive perseveration in rats. Neuropsychopharmacology 31: 757-767.

Carter ME, Soden ME, Zweifel LS, Palmiter RD (2013). Genetic identification of a neural circuit that suppresses appetite. Nature 503: 111-114.

Chen WJ, Faraone SV (2000). Sustained attention deficits as markers of genetic susceptibility to schizophrenia. Am J Med Genet 97: 52-57.

Chudasama Y, Passetti F, Rhodes SE, Lopian D, Desai A, Robbins TW (2003). Dissociable aspects of performance on the 5 -choice serial reaction time task following lesions of the dorsal anterior cingulate, infralimbic and orbitofrontal cortex in the rat: differential effects on selectivity, impulsivity and compulsivity. Behav Brain Res 146: 105-119.

Cohen RA, Kaplan RF, Moser DJ, Jenkins MA, Wilkinson H (1999). Impairments of attention after cingulotomy. Neurology 53: 819-824.

Corbetta M, Miezin FM, Dobmeyer S, Shulman GL, Petersen SE (1991). Selective and divided attention during visual discriminations of shape, color, and speed: functional anatomy by positron emission tomography. J Neurosci 11: 2383-2402.

Cornblatt BA, Malhotra AK (2001). Impaired attention as an endophenotype for molecular genetic studies of schizophrenia. Am J Med Genet 105: 11-15.

Cuthbert BN (2015). Research Domain Criteria: toward future psychiatric nosologies. Dialog Clin Neurosci 17: 89-97.

Demars MP, Morishita H (2014). Cortical parvalbumin and somatostatin GABA neurons express distinct endogenous modulators of nicotinic acetylcholine receptors. Mol Brain 7: 75 .

Fair DA, Dosenbach NU, Church JA, Cohen AL, Brahmbhatt S, Miezin FM et al (2007). Development of distinct control networks through segregation and integration. Proc Natl Acad Sci USA 104: 13507-13512.

Farrell MS, Roth BL (2013). Pharmacosynthetics: reimagining the pharmacogenetic approach. Brain Res 1511: 6-20.

Ferguson SM, Eskenazi D, Ishikawa M, Wanat MJ, Phillips PE, Dong $\mathrm{Y}$ et al (2011). Transient neuronal inhibition reveals opposing roles of indirect and direct pathways in sensitization. Nat Neurosci 14: 22-24.

Goodkind M, Eickhoff SB, Oathes DJ, Jiang Y, Chang A, Jones-Hagata LB et al (2015). Identification of a common neurobiological substrate for mental illness. JAMA Psychiatry 72: 305-315.

Homayoun H, Moghaddam B (2007). NMDA receptor hypofunction produces opposite effects on prefrontal cortex interneurons and pyramidal neurons. J Neurosci 27: 11496-11500.

Huang-Pollock CL, Karalunas SL, Tam H, Moore AN (2012). Evaluating vigilance deficits in ADHD: a meta-analysis of CPT performance. J Abnorm Psychol 121: 360-371.

Insel T, Cuthbert B, Garvey M, Heinssen R, Pine DS, Quinn K et al (2010). Research domain criteria (RDoC): toward a new classification framework for research on mental disorders. Am J Psychiatry 167: 748-751.

Johnston K, Levin HM, Koval MJ, Everling S (2007). Top-down control-signal dynamics in anterior cingulate and prefrontal cortex neurons following task switching. Neuron 53: 453-462.

Koch M, Varela L, Kim JG, Kim JD, Hernandez-Nuno F, Simonds SE et al (2015). Hypothalamic POMC neurons promote cannabinoid-induced feeding. Nature 519: 45-50.

Krashes MJ, Koda S, Ye C, Rogan SC, Adams AC, Cusher DS et al (2011). Rapid, reversible activation of AgRP neurons drives feeding behavior in mice. J Clin Invest 121: 1424-1428. 
Lazo OM, Mauna JC, Pissani CA, Inestrosa NC, Bronfman FC (2010). Axotomy-induced neurotrophic withdrawal causes the loss of phenotypic differentiation and downregulation of NGF signalling, but not death of septal cholinergic neurons. Mol Neurodegener 5: 5.

Li B, Wang L, Sun Z, Zhou Y, Shao D, Zhao J et al (2014). The anticonvulsant effects of SR 57227 on pentylenetetrazole-induced seizure in mice. PLoS One 9: e93158.

Lustig C, Kozak R, Sarter M, Young JW, Robbins TW (2013). CNTRICS final animal model task selection: control of attention. Neurosci Biobehav Rev 37: 2099-2110.

Mahler SV, Vazey EM, Beckley JT, Keistler CR, McGlinchey EM, Kaufling J et al (2014). Designer receptors show role for ventral pallidum input to ventral tegmental area in cocaine seeking. Nat Neurosci 17: 577-585.

Mar AC, Horner AE, Nilsson SR, Alsio J, Kent BA, Kim CH et al (2013). The touchscreen operant platform for assessing executive function in rats and mice. Nat Protoc 8: 1985-2005.

Moore H, Geyer MA, Carter CS, Barch DM (2013). Harnessing cognitive neuroscience to develop new treatments for improving cognition in schizophrenia: CNTRICS selected cognitive paradigms for animal models. Neurosci Biobehav Rev 37: 2087-2091.

Ochsner KN, Kosslyn SM, Cosgrove GR, Cassem EH, Price BH, Nierenberg AA et al (2001). Deficits in visual cognition and attention following bilateral anterior cingulotomy. Neuropsychologia 39: 219-230.

Parnaudeau S, O'Neill PK, Bolkan SS, Ward RD, Abbas AI, Roth BL et al (2013). Inhibition of mediodorsal thalamus disrupts thalamofrontal connectivity and cognition. Neuron 77: $1151-1162$.

Parnaudeau S, Taylor K, Bolkan SS, Ward RD, Balsam PD, Kellendonk C (2015). Mediodorsal thalamus hypofunction impairs flexible goal-directed behavior. Biol Psychiatry 77: 445-453.

Passetti F, Chudasama Y, Robbins TW (2002). The frontal cortex of the rat and visual attentional performance: dissociable functions of distinct medial prefrontal subregions. Cereb Cortex 12: 1254-1268.

Pehrson AL, Bondi CO, Totah NK, Moghaddam B (2013). The influence of NMDA and $\mathrm{GABA}(\mathrm{A})$ receptors and glutamic acid decarboxylase (GAD) activity on attention. Psychopharmacology 225: 31-39.

Penzo MA, Robert V, Tucciarone J, De Bundel D, Wang M, Van Aelst L et al (2015). The paraventricular thalamus controls a central amygdala fear circuit. Nature 519: 455-459.

Perova Z, Delevich K, Li B (2015). Depression of excitatory synapses onto parvalbumin interneurons in the medial prefrontal cortex in susceptibility to stress. J Neurosci 35: 3201-3206.

Ray RS, Corcoran AE, Brust RD, Kim JC, Richerson GB, Nattie E et al (2011). Impaired respiratory and body temperature control upon acute serotonergic neuron inhibition. Science 333: 637-642.

Richards BA, Xia F, Santoro A, Husse J, Woodin MA, Josselyn SA et al (2014). Patterns across multiple memories are identified over time. Nat Neurosci 17: 981-986.

Robbins TW (2002). The 5-choice serial reaction time task: behavioural pharmacology and functional neurochemistry. Psychopharmacology 163: 362-380.
Romberg C, Mattson MP, Mughal MR, Bussey TJ, Saksida LM (2011). Impaired attention in the $3 \times \mathrm{TgAD}$ mouse model of Alzheimer's disease: rescue by donepezil (Aricept). J Neurosci 31: 3500-3507.

Sasaki K, Suzuki M, Mieda M, Tsujino N, Roth B, Sakurai T (2011). Pharmacogenetic modulation of orexin neurons alters sleep/ wakefulness states in mice. PLoS One 6: e20360.

Silverman JL, Gastrell PT, Karras MN, Solomon M, Crawley JN (2013). Cognitive abilities on transitive inference using a novel touchscreen technology for mice. Cereb Cortex 25: 1133-1142.

Totah NK, Jackson ME, Moghaddam B (2013). Preparatory attention relies on dynamic interactions between prelimbic cortex and anterior cingulate cortex. Cereb Cortex 23: 729-738.

Totah NK, Kim YB, Homayoun H, Moghaddam B (2009). Anterior cingulate neurons represent errors and preparatory attention within the same behavioral sequence. J Neurosci 29: 6418-6426.

Urban DJ, Roth BL (2015). DREADDs (designer receptors exclusively activated by designer drugs): chemogenetic tools with therapeutic utility. Annu Rev Pharmacol Toxicol 55: 399-417.

Uylings HB, Groenewegen HJ, Kolb B (2003). Do rats have a prefrontal cortex? Behav Brain Res 146: 3-17.

Vogt BA, Paxinos G (2014). Cytoarchitecture of mouse and rat cingulate cortex with human homologies. Brain Struct Funct 219: 185-192.

Weissman DH, Gopalakrishnan A, Hazlett CJ, Woldorff MG (2005). Dorsal anterior cingulate cortex resolves conflict from distracting stimuli by boosting attention toward relevant events. Cereb Cortex 15: 229-237.

Young JW, Amitai N, Geyer MA (2012). Behavioral animal models to assess pro-cognitive treatments for schizophrenia. Handb Exp Pharmacol 39-79.

Young JW, Geyer MA (2015). Developing treatments for cognitive deficits in schizophrenia: the challenge of translation. J Psychopharmacol 29: 178-196.

Young JW, Geyer MA, Rissling AJ, Sharp RF, Eyler LT, Asgaard GL et al (2013a). Reverse translation of the rodent 5C-CPT reveals that the impaired attention of people with schizophrenia is similar to scopolamine-induced deficits in mice. Transl Psychiatry 3: e324.

Young JW, Jentsch JD, Bussey TJ, Wallace TL, Hutcheson DM (2013b). Consideration of species differences in developing novel molecules as cognition enhancers. Neurosci Biobehav Rev 37: 2181-2193.

Young JW, Light GA, Marston HM, Sharp R, Geyer MA (2009). The 5-choice continuous performance test: evidence for a translational test of vigilance for mice. PLoS One 4: e4227.

Zhang S, Xu M, Kamigaki T, Hoang Do JP, Chang WC, Jenvay S et al (2014). Selective attention. Long-range and local circuits for top-down modulation of visual cortex processing. Science 345: 660-665.

Zhu H, Roth BL (2014). DREADD: a chemogenetic GPCR signaling platform. Int J Neuropsychopharmacol 18 Please provide the page range in reference Zhu and Roth, 2014..

Zingg B, Hintiryan H, Gou L, Song MY, Bay M, Bienkowski MS et al (2014). Neural networks of the mouse neocortex. Cell 156: 1096-1111.

Supplementary Information accompanies the paper on the Neuropsychopharmacology website (http://www.nature.com/npp) 